\title{
Observer Design for One-Sided Lipschitz Nonlinear Systems Subject to Measurement Delays
}

\author{
Sohaira Ahmad, ${ }^{1}$ Raafia Majeed, ${ }^{1}$ Keum-Shik Hong, ${ }^{2}$ and Muhammad Rehan ${ }^{1}$ \\ ${ }^{1}$ Department of Electrical Engineering, Pakistan Institute of Engineering and Applied Sciences (PIEAS), \\ P.O. Box 45650, Islamabad, Pakistan \\ ${ }^{2}$ School of Mechanical Engineering, Pusan National University, 2 Busandaehak-ro, Geumjeong-gu, Busan 609-735, Republic of Korea
}

Correspondence should be addressed to Muhammad Rehan; rehanqau@gmail.com

Received 6 June 2014; Revised 11 August 2014; Accepted 11 August 2014

Academic Editor: Yuxin Zhao

Copyright (C) 2015 Sohaira Ahmad et al. This is an open access article distributed under the Creative Commons Attribution License, which permits unrestricted use, distribution, and reproduction in any medium, provided the original work is properly cited.

\begin{abstract}
This paper presents a novel nonlinear observer-design approach to one-sided Lipschitz nonlinear systems in the presence of output delays. The crux of the approach is to overcome the practical consequences of time delays, encountered due to distant sensor position and time lag in measurement, for estimation of physical and engineering nonlinear system states. A Lyapunov-Krasovskii functional is employed, the time derivative of which is solved using Jensen's inequality, one-sided Lipschitz condition, and quadratic innerboundedness, and, accordingly, design conditions for delay-range-dependent nonlinear observer for delayed one-sided Lipschitz systems are derived. Further, novel solutions to the problems of delay-dependent observer synthesis of one-sided Lipschitz models and delay-range-dependent state estimation of linear and Lipschitz nonlinear systems are deduced from the present delay-rangedependent technique. An observer formulation methodology for retrieval of one-sided Lipschitz nonlinear-system states, which is robust against $L_{2}$ norm-bounded perturbations, is devised. The resultant design conditions, in contrast to the conventional procedures, can be solved via less conservative linear matrix inequality- (LMI-) based routines that succeed by virtue of additional LMI variables, meaningful transformations, and cone complementary linearization algorithm. Numerical examples are worked out to illustrate the effectiveness of the proposed observer-synthesis approach for delayed one-sided Lipschitz systems.
\end{abstract}

\section{Introduction}

State estimation using an observer is a methodology widely employed in physical, biomedical, and engineering fields owing to its multitude of applications in road-gradient and vehicle-mass estimation, coestimation for lithium-polymer battery cells, online monitoring of nonlinear bioprocesses, identification and analysis of vascular tumor growth, detection and reconstruction of sensor faults, robust control of stochastic systems under disturbances, and cylinder-pressure reconstruction [1-7]. Observer synthesis for nonlinear systems has received considerable attention within the control field over the past few decades, as it makes possible the application of state estimation to control design, energy system analysis, fault diagnosis, chaos-based secure communications, synchronization studies, and unknown input recovery [8-11]. Notable work in this regard has been concentrated on continuous-time systems, while a certain quantity of research has been devoted to discrete-time and time-delay dynamical models [12-15]. For the nonlinear systems, the observer design remains a challenging research problem: no generalized or widely applicable solution has yet been reported or even explored. Nonlinear observer design in the presence of time delays is thought-provoking, particularly as sensor technologies, conditioning units, and measurement systems often introduce unavoidable time delays that can sabotage the practicality of a monitoring or a control system [16-18].

In the literature, two broad and widely employed methodologies for observer design of nonlinear systems are nonlinear state transformation, for which the state-estimation error dynamics are transformed into linear ones $[19,20]$, and the direct method, based on the original system, by which the estimation error dynamics are obtained in nonlinear form [21-23]. Thus far, for the class of nonlinear systems observing the Lipschitz condition (see, e.g., [24] and references therein), 
multiple observer designs based on the direct method have been presented to address the existence condition for fullorder and reduced-order observers, robustness in observer design subject to disturbances, robust sensor fault reconstruction, and observer synthesis for discrete-time systems [25-28]. The Lipschitz condition in numerical analysis and mathematics, found to be conservative and region based, is now being replaced by a more spacious and less conservative one-sided Lipschitz condition. Observer-design schemes, based on characteristics of Lipschitz functions, ensure stability of state-estimation error only for small values of Lipschitz constants and result into infeasibility if the aforesaid constant is large. These facts escalate the demand of the one-sided Lipschitz constant for approximating an upper bound on nonlinear component of a dynamical system to accomplish viable estimation of the full state vector.

In recent years, several observer-design problems for one-sided Lipschitz nonlinear systems have been investigated [29-32]. For example, a state observer-design scheme for discrete-time systems with mathematical artifacts on the Lyapunov function for obtainment of simple linear matrix inequality (LMI) conditions for asymptotic stability of state-estimation error was carried out [29]. Full-order and reduced-order observer designs for one-sided Lipschitz systems using the Riccati equation approach demonstrating less conservatism than the Lipschitz counterpart also have been studied [30]. Further, a methodology applicable to monotonic and Lipschitz as well as one-sided Lipschitz nonlinearities was presented, by which the observer gain matrix is determined by solving LMIs [31]. In another approach, the analysis and deduction problem in a unified LMI framework, which provides the condition for existence of a nonlinear state observer, is addressed by incorporating the concept of quadratic inner-boundedness [32]. Still, however, for delayed one-sided Lipschitz nonlinear systems, asymptotic stability conditions to the observer-design dilemma remain elusive owing to the twofold involvement of time delays and onesided Lipschitz dynamics. In this regard, the presence of output delays in practical systems, unavoidable due to the distant sensor position, digital processor computations, and measurement system processing, can result in oscillations, lags, and even instability, making the traditional observerdesign approaches like [29-32] infeasible for state estimation. Moreover, the one-sided Lipschitz constant is either significantly smaller than or at most equal to the traditional Lipschitz constant, which fact facilitates a more suitable observer construction of nonlinear time-delay systems by reckoning the influence of nonlinear and delayed nonlinear parts. If observer-design techniques can be developed for delayed one-sided Lipschitz systems, these schemes can be effectively utilized or reformulated for monitoring and control of complex forms of engineering systems.

Time delays, varying in an interval [33] and appearing in state, input, and output variables as well as in state derivatives, are frequently encountered in engineering and physical systems [33-38]. In recent years, the stability analysis and control design problems were investigated for time-variant and timeinvariant delayed systems (see, for instance, reference [39, 40]). Delay-dependent stability approach assuming that the time delay belongs to an interval from zero to a constant value provides less conservative results than does the conventional delay-independent scheme [41]. Nevertheless, because the Lyapunov function ignores the lower bound of the time delay, conservatism remains; therefore, the lower bound should be incorporated to establish less restricted results. Recently, delay-range-dependent techniques addressing the problem of conservatism have been developed (see, e.g., [33, 42-45]) for linear time-delay systems, based on various LyapunovKrasovskii (LK) approaches, Jensen's inequality, the freeweighting matrix, Newton Leibniz, and others; however, the field of delay-range-dependent observer synthesis for linear and, particularly, for nonlinear time-delay systems is still relatively immature. The beauty of such delay-rangedependent state-estimation methodologies lies in their applicability to systems with either large or small delays; therefore, it will be interesting to explore a state-of-the-art delayrange-dependent observer-design strategy for the one-sided Lipschitz nonlinear systems with measurement delays.

Motivated by the aforementioned linear delay-rangedependent approaches and one-sided Lipschitz nonlinear observer construction methodologies, the present study explores the problem of a nonlinear observer design for one-sided Lipschitz nonlinear systems under outputmeasurement and processing delays. By application of an LK functional, the time derivative of which is solved using Jensen's inequality, incorporating the one-sided Lipschitz condition and quadratic inner-boundedness, exploiting the standard matrix inequality procedures and regarding the nonzero lower bound of the output delay, a delay-range-dependent Luenberger-type observer-synthesis scheme ensuring asymptotic estimation of the states of delayed one-sided Lipschitz nonlinear plants was established. To the best of the authors' knowledge, the proposed observerdesign scheme enabling time-delay one-sided Lipschitz nonlinear systems to overcome the practical limitation of sensors and measurement systems is herein introduced for the first time. The more general feature of the proposed one-sided Lipschitz observer-synthesis treatment relative to the Lipschitz one for the case of output delays is addressed by establishing a relation, and no such delay-range-dependent results for observer formulation using the Lipschitz condition have yet been effectively reported. Furthermore, a delay-dependent approach is deduced as a particular case of the present delayrange-dependent observer-design methodology, by taking the zero value of the lower bound on the time delay. A delayrate-independent condition, owing to its importance in the field of full state vector estimation, is provided by application of the proposed LK functional treatment.

The scope of the resultant observer-design study for nonlinear interval time-delay systems is extended to fast time-varying delays, and the corresponding conditions are detailed. In addition, robust observer-synthesis scheme in the presence of output delays is provided against $L_{2}$ normbounded perturbations and disturbances to guarantee $L_{2}$ gain reduction from unwanted signals to the state-estimation error. Another contribution of the present work is the formulation of a less conservative LMI-based routine compared with the delay-range-dependent approaches like [46], 
obtained by introducing additional LMI variables and meaningful transformations, solvable via a cone complementary linearization algorithm. Two numerical simulation examples are provided to illustrate the effectiveness of the proposed observer-design methodology for delayed one-sided Lipschitz nonlinear systems.

The remainder of the paper is organized as follows. In Section 2, the problem is formulated and some important concepts are introduced. Section 3 presents the main design conditions for the delay-range-dependence-oriented nonlinear dynamical observer strategies and, further, discusses their various modifications. Sections 4 and 5 provide simulations and concluding remarks, respectively.

Standard notation is used throughout the paper. The Euclidean norm of a vector $x$ is shown by $\|x\|$, and the $L_{2}$ norm of the vector by $\|x\|_{2}=\sqrt{\int_{0}^{\infty}\|x\|^{2} d t}$. For vectors $x$ and $y$ of the same dimension, the notation $\langle x, y\rangle$ represents the inner product of the vectors. The mathematical quantity $\sup _{\|d\|_{2} \neq 0}\left(\|e\|_{2} /\|d\|_{2}\right)$ defines the $L_{2}$ gain for a system with an input vector $d$ and an output vector $e$. A symmetric positive (or semipositive) definite matrix $X$ is stated by matrix inequality $X>0$ (or $X \geq 0$ ). The transpose of a matrix $A$ is noted as $A^{T}$. The notation $\operatorname{diag}\left(x_{1}, x_{2}, \ldots, x_{n}\right)$ denotes a block diagonal matrix with entry $x_{i}$, for $i=1,2, \ldots, n$, at the corresponding diagonal entry.

\section{System Description}

Consider a class of one-sided Lipschitz nonlinear dynamical systems with time-varying output delays, given by

$$
\begin{gathered}
\dot{x}(t)=A x(t)+f(x, u)+d, \\
y(t)=C x(t-\tau),
\end{gathered}
$$

where $x \in \mathfrak{R}^{n}, u \in \Re^{m}, y \in \mathfrak{R}^{p}$, and $d \in \mathfrak{R}^{n}$ are the state vector, the control input, the output, and the disturbance to the system, respectively. The linear constant matrices of the dynamical system are represented by $A$ and $C$, and the nonlinear function is denoted by $f(x, u) \in \mathfrak{R}^{n}$. A continuous time-varying differentiable function $\tau$ refers to the time delay at the output, satisfying

$$
\begin{gathered}
0 \leq h_{1} \leq \tau \leq h_{2}, \\
\dot{\tau} \leq \mu .
\end{gathered}
$$

The function $f(x, u)$ belongs to the one-sided Lipschitz nonlinearities owing to the definition given below (see, for instance, [29-32]). Another concept employed for the observer design is quadratic inner-boundedness (see $[30,32$, 47]).

Definition 1. A nonlinear function $f(x, u)$ is said to be onesided Lipschitz in a region $D$ enclosing the origin if there exists a scalar $\rho \in \mathfrak{R}$ such that the relation

$$
\langle f(x, u)-f(\bar{x}, u), x-\bar{x}\rangle \leq \rho\|x-\bar{x}\|^{2},
$$

holds $\forall x, \bar{x} \in D$, where $\rho$ is the one-sided Lipschitz constant.
Definition 2. A nonlinear function $f(x, u)$ is said to satisfy the quadratic inner-boundedness condition in a defined region $D$, if there exist scalars $\beta, \alpha \in \mathfrak{R}$, such that

$$
\begin{aligned}
& (f(x, u)-f(\bar{x}, u))^{T}(f(x, u)-f(\bar{x}, u)) \\
& \quad \leq \beta\|x-\bar{x}\|^{2}+\alpha\langle x-\bar{x}, f(x, u)-f(\bar{x}, u)\rangle
\end{aligned}
$$

is satisfied for all $x, \bar{x} \in D$.

The one-sided Lipschitz and quadratic inner-boundedness conditions extrapolate the definitive Lipschitz theory to a more ecumenical category of nonlinear systems and have inbuilt advantages in observer synthesis. For a given function $f(x, u)$ satisfying (3)-(4), the Lipschitz condition holds, whereas the reverse is not true (see details in [30, 32, 47]). Further, the one-sided Lipschitz constant $\rho$ and the quadratic inner-boundedness parameter $\beta$ can be any real numbers, unlike the Lipschitz constant, which needs to be always positive.

The aim of the present study is to propose an observerdesign methodology for a dynamic one-sided Lipschitz nonlinear system (1) subject to time-varying output delays belonging to an interval.

\section{Observer Design}

Consider a Luenberger-like observer for a delayed one-sided Lipschitz nonlinear system (1) formulated as

$$
\begin{gathered}
\dot{\hat{x}}(t)=A \widehat{x}(t)+f(\widehat{x}, u)+L(y(t)-\widehat{y}(t)), \\
\hat{y}(t)=C \hat{x}(t-\tau),
\end{gathered}
$$

where $L \in \mathfrak{R}^{n \times m}$ is the observer gain matrix. The stateestimation error is given by

$$
e=x-\widehat{x}
$$

From (1) and (5)-(6), we have the error dynamics:

$$
\dot{e}(t)=A e(t)+f(x, u)-f(\widehat{x}, u)-L(y(t)-\widehat{y}(t))+d,
$$

which reduce further to

$$
\dot{e}(t)=A e(t)+\Phi(x, \widehat{x}, u)-L C e(t-\tau)+d,
$$

by substitution of

$$
\Phi(x, \widehat{x}, u)=f(x, u)-f(\widehat{x}, u)
$$

Now, we provide an LMI-based sufficient condition to test the state-estimation ability of an observer (5) for a given observer gain matrix $L$. Note that a sophisticated guess of the observer gain matrix can be obtained using the traditional observer-design methodologies such as the standard highgain-observer approach and techniques (see, e.g., [10, 29-32], etc.) that do not involve time delays. 
Theorem 3. Consider a one-sided Lipschitz nonlinear system (1) satisfying the time-delay bounds given by (2), the one-sided Lipschitz condition (3), and the quadratic inner-boundedness criterion (4). Suppose there exist symmetric matrices $P \in$ $\mathfrak{R}^{n \times n}, Q_{i} \in \mathfrak{R}^{n \times n}$, and $Z_{j} \in \mathfrak{R}^{n \times n}$ for $i=1,2,3$, and $j=1,2$, and scalars $\varepsilon_{1}$ and $\varepsilon_{2}$, such that the LMIs

$$
\begin{gathered}
P>0, \quad Q_{i}>0, \\
{\left[\begin{array}{ccccccc}
Y_{1}+\rho \varepsilon_{1} I+\beta \varepsilon_{2} I & -P L C & Z_{1} & 0 & P-\frac{\varepsilon_{1} I}{2}+\frac{\alpha \varepsilon_{2} I}{2} & h_{1} A^{T} Z_{1} & h_{12} A^{T} Z_{2} \\
* & -\Lambda_{1} & Z_{2} & Z_{2} & 0 & -h_{1} C^{T} L^{T} Z_{1} & -h_{12} C^{T} L^{T} Z_{2} \\
* & * & -\Lambda_{2} & 0 & 0 & 0 & 0 \\
* & * & * & -\Lambda_{3} & 0 & h_{1} Z_{1} & h_{12} Z_{2} \\
* & * & * & * & -\varepsilon_{2} I & h_{1} Z_{1} & h_{12} Z_{2} \\
* & * & * & * & * & -Z_{1} & 0 \\
* & * & * & * & * & * & -Z_{2}
\end{array}\right]<0}
\end{gathered}
$$

are satisfied for a given matrix $L$, where

$$
\begin{gathered}
Y_{1}=P A+A^{T} P+\sum_{i=1}^{3} Q_{i}-Z_{1}, \\
\Lambda_{1}=(1-\mu) Q_{3}+2 Z_{2}, \\
\Lambda_{2}=Q_{1}+Z_{1}+Z_{2}, \\
\Lambda_{3}=Q_{2}+Z_{2}, \\
h_{12}=h_{2}-h_{1} .
\end{gathered}
$$

Then, there exists a Luenberger-type observer (5) such that the state-estimation error e asymptotically converges to the origin.

Proof. Define an LK functional candidate (see, for instance, [43]) as

$$
\begin{aligned}
V(e, t)= & e^{T} P e+\sum_{i=1}^{2} \int_{t-h_{i}}^{t} e^{T}(\alpha) Q_{i} e(\alpha) d \alpha \\
& +\int_{t-\tau}^{t} e^{T}(\alpha) Q_{3} e(\alpha) d \alpha \\
& +\int_{-h_{1}}^{0} \int_{t+s}^{t} h_{1} \dot{e}^{T}(\alpha) Z_{1} \dot{e}(\alpha) d \alpha d s \\
& +\int_{-h_{2}}^{-h_{1}} \int_{t+s}^{t} h_{12} \dot{e}^{T}(\alpha) Z_{2} \dot{e}(\alpha) d \alpha d s
\end{aligned}
$$

Acquiring the time derivative of (13) yields

$$
\begin{aligned}
\dot{V}(e, t)= & 2 e^{T} P \dot{e}+\sum_{i=1}^{2}\left\{e^{T} Q_{i} e-e^{T}\left(t-h_{i}\right) Q_{i} e\left(t-h_{i}\right)\right\} \\
& +e^{T} Q_{3} e-(1-\mu) e^{T}(t-\tau) Q_{3} e(t-\tau) \\
& +\dot{e}^{T}\left(h_{1}^{2} Z_{1}+h_{12}^{2} Z_{2}\right) \dot{e}-\int_{t-h_{1}}^{t} h_{1} \dot{e}^{T}(\alpha) Z_{1} \dot{e}(\alpha) d \alpha \\
& -\int_{t-h_{2}}^{t-h_{1}} h_{12} \dot{e}^{T}(\alpha) Z_{2} \dot{e}(\alpha) d \alpha .
\end{aligned}
$$

Employing (8) and (14) and rearranging the terms, the upper bound on $\dot{V}(e, t)$ is obtained as

$$
\begin{aligned}
\dot{V}(e, t) \leq & 2 e^{T} P(A e+\Phi(x, \widehat{x}, u)+d-L C e(t-\tau)) \\
& +\sum_{i=1}^{3} e^{T} Q_{i} e-\sum_{i=1}^{2} e^{T}\left(t-h_{i}\right) Q_{i} e\left(t-h_{i}\right) \\
& -(1-\mu) e^{T}(t-\tau) Q_{3} e(t-\tau) \\
& -\int_{t-h_{1}}^{t} h_{1} \dot{e}^{T}(\alpha) Z_{1} \dot{e}(\alpha) d \alpha \\
& +(A e+\Phi(x, \widehat{x}, u)+d-L C e(t-\tau))^{T} \\
& \times\left(h_{1}^{2} Z_{1}+h_{12}^{2} Z_{2}\right) \\
& \times(A e+\Phi(x, \widehat{x}, u)+d-L C e(t-\tau)) \\
& -\int_{t-h_{2}}^{t-h_{1}} h_{12} \dot{e}^{T}(\alpha) Z_{2} \dot{e}(\alpha) d \alpha .
\end{aligned}
$$

Applying Jensen's inequality reveals

$$
\begin{aligned}
&-\int_{t-h_{1}}^{t} h_{1} \dot{e}^{T}(\alpha) Z_{1} \dot{e}(\alpha) d \alpha \\
& \quad \leq-\left(\int_{t-h_{1}}^{t} \dot{e}(\alpha) d \alpha\right)^{T} Z_{1}\left(\int_{t-h_{1}}^{t} \dot{e}(\alpha) d \alpha\right) \\
& \quad \leq-\left(e(t)-e\left(t-h_{1}\right)\right)^{T} Z_{1}\left(e(t)-e\left(t-h_{1}\right)\right) .
\end{aligned}
$$

Similarly, we have

$$
\begin{aligned}
& -\int_{t-h_{2}}^{t-h_{1}} h_{12} \dot{e}^{T}(\alpha) Z_{2} \dot{e}(\alpha) d \alpha \\
& =-\int_{t-h_{2}}^{t-\tau} h_{12} \dot{e}^{T}(\alpha) Z_{2} \dot{e}(\alpha) d \alpha \\
& -\int_{t-\tau}^{t-h_{1}} h_{12} \dot{e}^{T}(\alpha) Z_{2} \dot{e}(\alpha) d \alpha,
\end{aligned}
$$




$$
\begin{aligned}
-\int_{t-h_{2}}^{t-h_{1}} h_{12} \dot{e}^{T}(\alpha) Z_{2} \dot{e}(\alpha) d \alpha & \times\left[-(1-\mu) Q_{3}-2 Z_{2}+C^{T} L^{T}\left(h_{1}^{2} Z_{1}+h_{12}^{2} Z_{2}\right) L C\right] \\
\leq-\left(\int_{t-h_{2}}^{t-\tau} \dot{e}(\alpha) d \alpha\right)^{T} Z_{2}\left(\int_{t-h_{2}}^{t-\tau} \dot{e}(\alpha) d \alpha\right) & \times e(t-\tau)+2 e^{T}(t-\tau) Z_{2} e\left(t-h_{1}\right)+2 e^{T}(t-\tau) \\
-\left(\int_{t-\tau}^{t-h_{1}} \dot{e}(\alpha) d \alpha\right)^{T} Z_{2}\left(\int_{t-\tau}^{t-h_{1}} \dot{e}(\alpha) d \alpha\right), & \times Z_{2} e\left(t-h_{2}\right)+e^{T}\left(t-h_{1}\right)\left(-Q_{1}-Z_{1}-Z_{2}\right) e\left(t-h_{1}\right) \\
-\int_{t-h_{2}}^{t-h_{1}} h_{12} \dot{e}^{T}(\alpha) Z_{2} \dot{e}(\alpha) d \alpha & +e^{T}\left(t-h_{2}\right)\left(-Q_{2}-Z_{2}\right) e\left(t-h_{2}\right) \\
\leq-\left(e(t-\tau)-e\left(t-h_{2}\right)\right)^{T} Z_{2}\left(e(t-\tau)-e\left(t-h_{2}\right)\right) & +2 e^{T}\left[P+A^{T}\left(h_{1}^{2} Z_{1}+h_{12}^{2} Z_{2}\right)\right] \Phi(x, \widehat{x}, u) \\
-\left(e\left(t-h_{1}\right)-e(t-\tau)\right)^{T} Z_{2}\left(e\left(t-h_{1}\right)-e(t-\tau)\right) . & +2 e^{T}\left[P+A^{T}\left(h_{1}^{2} Z_{1}+h_{12}^{2} Z_{2}\right)\right] d+\Phi^{T}(x, \widehat{x}, u) \\
& \times\left(h_{1}^{2} Z_{1}+h_{12}^{2} Z_{2}\right) \Phi(x, \widehat{x}, u)+2 \Phi^{T}(x, \widehat{x}, u) \\
& \times\left(h_{1}^{2} Z_{1}+h_{12}^{2} Z_{2}\right) d+d^{T}\left(h_{1}^{2} Z_{1}+h_{12}^{2} Z_{2}\right) d \\
\dot{V}(e, t) & -2 d^{T}\left(h_{1}^{2} Z_{1}+h_{12}^{2} Z_{2}\right) L C e(t-\tau) \\
& -2 e^{T}(t-\tau)\left(C^{T} L^{T}\left(h_{1}^{2} Z_{1}+h_{12}^{2} Z_{2}\right)\right) \Phi(x, \widehat{x}, u) .
\end{aligned}
$$

$$
\begin{aligned}
\leq & e^{T}\left[P A+A^{T} P+\sum_{i=1}^{3} Q_{i}+A^{T}\left(h_{1}^{2} Z_{1}+h_{12}^{2} Z_{2}\right) A-Z_{1}\right] e \\
& -2 e^{T}\left[P L C+A^{T}\left(h_{1}^{2} Z_{1}+h_{12}^{2} Z_{2}\right) L C\right] e(t-\tau) \\
& +2 e^{T} Z_{1} e\left(t-h_{1}\right)+e^{T}(t-\tau)
\end{aligned}
$$$$
\text { Under } d=0 \text {, (18) implies }
$$$$
\dot{V}(e, t) \leq \Psi_{1}^{T} \Upsilon_{1} \Psi_{1}
$$

where

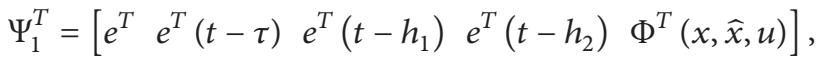

$$
\begin{aligned}
& \Upsilon_{1}=\left[\begin{array}{ccccc}
Y_{1}+A^{T} Y_{4} A & -P L C-A^{T} Y_{4} L C & Z_{1} & 0 & Y_{2} \\
* & -(1-\mu) Q_{3}-2 Z_{2}+Y_{3} L C & Z_{2} & Z_{2} & -Y_{3} \\
* & * & -Q_{1}-Z_{1}-Z_{2} & 0 & 0 \\
* & * & * & -Q_{2}-Z_{2} & 0 \\
* & * & * & * & Y_{4}
\end{array}\right]<0 \text {, } \\
& Y_{2}=P+A^{T}\left(h_{1}^{2} Z_{1}+h_{12}^{2} Z_{2}\right) \\
& Y_{3}=C^{T} L^{T}\left(h_{1}^{2} Z_{1}+h_{12}^{2} Z_{2}\right) \\
& Y_{4}=\left(h_{1}^{2} Z_{1}+h_{12}^{2} Z_{2}\right)
\end{aligned}
$$

The one-sided Lipschitz condition given by (3) is equivalent to $\rho e^{T} e-e^{T} \Phi \geq 0$. For a positive scalar $\varepsilon_{1}$, the expression can be written as

$$
\Psi_{1}^{T}\left[\begin{array}{ccccc}
\rho \varepsilon_{1} I & 0 & 0 & 0 & \frac{-\varepsilon_{1} I}{2} \\
0 & 0 & 0 & 0 & 0 \\
0 & 0 & 0 & 0 & 0 \\
0 & 0 & 0 & 0 & 0 \\
\frac{-\varepsilon_{1} I}{2} & 0 & 0 & 0 & 0
\end{array}\right] \Psi_{1} \geq 0
$$

The quadratic inner-boundedness condition implies $\Phi^{T} \Phi \leq$ $\beta e^{T} e+\alpha e^{T} \Phi$, which for a positive scalar $\varepsilon_{2}$ results in

$$
\Psi_{1}^{T}\left[\begin{array}{ccccc}
\beta \varepsilon_{2} I & 0 & 0 & 0 & \frac{\alpha \varepsilon_{2} I}{2} \\
0 & 0 & 0 & 0 & 0 \\
0 & 0 & 0 & 0 & 0 \\
0 & 0 & 0 & 0 & 0 \\
\frac{\alpha \varepsilon_{2} I}{2} & 0 & 0 & 0 & -\varepsilon_{2} I
\end{array}\right] \Psi_{1} \geq 0
$$


Merging (21), (23), and (24) using the $S$-procedure entails

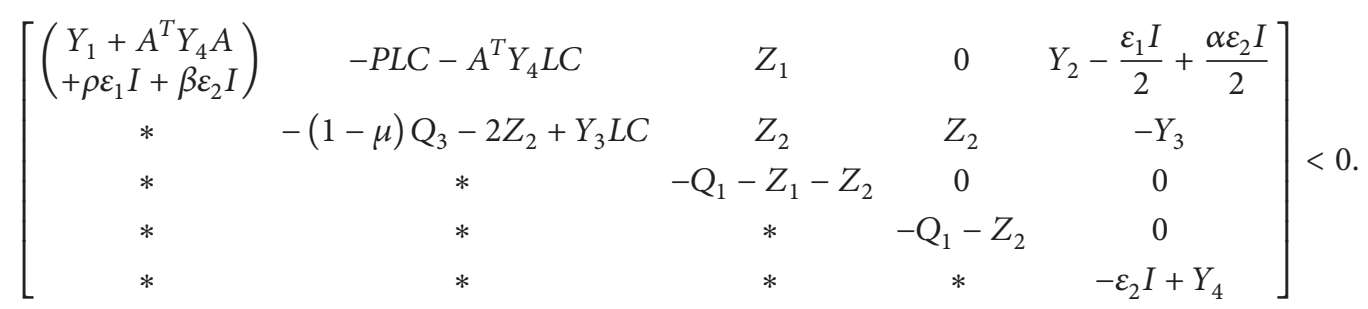

Applying the Schur complement (see, e.g., [35]) to (25) produces (11), which implies that $\dot{V}(e, t) \leq \Psi_{1}^{T} \Upsilon_{1} \Psi_{1}<0$; that is, the error $e$ asymptotically converges to the origin. This finishes the proof of Theorem 3.

Theorem 3 ensures state estimation by means of an observer for a given gain matrix $L$. If a guess for the observer gain matrix $L$ is unobtainable, the following Theorem 4 provides a solution.
Theorem 4. Consider a one-sided Lipschitz nonlinear system (1) satisfying the time-delay bounds given by (2), the one-sided Lipschitz condition (3), and the quadratic inner-boundedness criterion (4). Suppose there exist symmetric matrices $P \in$ $\mathfrak{R}^{n \times n}, Q_{i} \in \mathfrak{R}^{n \times n}$, and $Z_{j} \in \mathfrak{R}^{n \times n}$ for $i=1,2,3$, and $j=1,2$, matrix $X \in \mathfrak{R}^{n \times m}$, and scalars $\varepsilon_{1}$ and $\varepsilon_{2}$, such that the inequalities (10) and

$$
\left[\begin{array}{ccccccc}
Y_{1}+\rho \varepsilon_{1} I+\beta \varepsilon_{2} I & -X C & Z_{1} & 0 & P-\frac{\varepsilon_{1} I}{2}+\frac{\alpha \varepsilon_{2} I}{2} & h_{1} A^{T} P & h_{12} A^{T} P \\
* & -\Lambda_{1} & Z_{2} & Z_{2} & 0 & -h_{1} C^{T} X^{T} & -h_{12} C^{T} X^{T} \\
* & * & -\Lambda_{2} & 0 & 0 & 0 & 0 \\
* & * & * & -\Lambda_{3} & 0 & h_{1} P & h_{12} P \\
* & * & * & * & -\varepsilon_{2} I & h_{1} P & h_{12} P \\
* & * & * & * & * & -T_{1} & 0 \\
* & * & * & * & * & * & -T_{2}
\end{array}\right]<0
$$

are satisfied, where $T_{1}=P Z_{1}^{-1} P$ and $T_{2}=P Z_{2}^{-1} P$. Then, there exists a Luenberger-type observer (5) such that the stateestimation error e asymptotically converges to the origin.

Proof. Employing the congruence transform using $\operatorname{diag}(I, I$, $I, I, I, P Z_{1}^{-1}, P Z_{2}^{-1}$ ) to the inequality (11) and defining $X=P L$ and $T_{i}=P Z_{i}^{-1} P$ for $i=1,2$, we obtain (26). This completes the proof of Theorem 4 .

Remark 5. One-sided Lipschitz nonlinear observer designs for output-delay systems are presented in Theorems 3 and 4 by incorporating the quadratic inner-boundedness condition and the one-sided Lipschitz constraint. It should be emphasized that the proposed approach, in contrast to the approaches in [29-32] considering the delay free systems, fills the research gap in observer synthesis for one-sided Lipschitz nonlinear output-delay systems. It is also notable that the estimation results for the full state vector of the onesided Lipschitz nonlinear systems, in any form of time delay, are lacking in the literature. The presented approach in the present study can be further unfolded to the state estimation of nonlinear dynamic systems under delays in the states.

Remark 6. The projected observer-design methodology provided in Theorems 3 and 4 can be applied to a broad category of nonlinear systems for two reasons. First, it leads to a more general feature of one-sided Lipschitz nonlinearity compared with the Lipschitz one that guarantees state estimation for a relatively larger category of nonlinear systems. Second, the proposed observer scheme has been inferred for time-varying time-delay systems that satisfy the interval $0 \leq h_{1} \leq \tau \leq h_{2}$, which commonly is not addressed. Consequently, the presented delay-rangedependent observer-synthesis methodology is less conservative and more pragmatic than the traditional approaches such as $[16,17,23-32,34]$.

The proposed delay-range-dependent nonlinear observer-design scheme renders constructive linear results, as in a particular case $f(x, u)=B u$ below (see Corollary 7). The novel delay-range-dependent Lipschitz observer-synthesis scheme can also be deduced from the one-sided Lipschitz case (see Corollary 8) by taking a specific form of the quadratic inner-boundedness constants given by $\beta=l^{2}$ and $\alpha=0$, where $l$ is the Lipschitz constant for $f(x, u)$.

Corollary 7. Consider a linear system (1) with $f(x, u)=$ $B u$ (where $B$ is a constant input matrix of an appropriate dimension) satisfying the time-delay bounds given by (2). Suppose there exist symmetric matrices $P \in \mathfrak{R}^{n \times n}, Q_{i} \in \mathfrak{R}^{n \times n}$, 
and $Z_{j} \in \mathfrak{R}^{n \times n}$ for $i=1,2,3$, and $j=1,2$, and a matrix $X \in \mathfrak{R}^{n \times m}$ such that the inequalities

$$
\begin{gathered}
P>0, \quad Q_{i}>0, \\
{\left[\begin{array}{cccccc}
Y_{1} & -X C & Z_{1} & 0 & h_{1} A^{T} P & h_{12} A^{T} P \\
* & -\Lambda_{1} & Z_{2} & Z_{2} & -h_{1} C^{T} X^{T} & -h_{12} C^{T} X^{T} \\
* & * & -\Lambda_{2} & 0 & 0 & 0 \\
* & * & * & -\Lambda_{3} & 0 & 0 \\
* & * & * & * & -T_{1} & 0 \\
* & * & * & * & * & -T_{2}
\end{array}\right]<0}
\end{gathered}
$$

are satisfied. Then, there exists a Luenberger-type observer (5) for $f(\widehat{x}, u)=B u$ with observer gain matrix $L=P^{-1} X$ such that the state-estimation error e asymptotically converges to the origin.

Corollary 8. Consider a Lipschitz nonlinear system (1) satisfying the time-delay bounds given by (2) and the quadratic innerboundedness criterion (4) for $\beta=l^{2}$ and $\alpha=0$, where $l$ is the Lipschitz constant for $f(x, u)$. Suppose there exist symmetric matrices $P \in \mathfrak{R}^{n \times n}, Q_{i} \in \mathfrak{R}^{n \times n}$, and $Z_{j} \in \mathfrak{R}^{n \times n}$ for $i=1,2,3$, and $j=1,2$, matrix $X \in \mathfrak{R}^{n \times m}$, and a scalar $\varepsilon_{2}$, such that the inequalities (27), $\varepsilon_{2}>0$, and

$$
\left[\begin{array}{ccccccc}
Y_{1}+\varepsilon_{2} l^{2} I & -X C & Z_{1} & 0 & P & h_{1} A^{T} P & h_{12} A^{T} P \\
* & -\Lambda_{1} & Z_{2} & Z_{2} & 0 & -h_{1} C^{T} X^{T} & -h_{12} C^{T} X^{T} \\
* & * & -\Lambda_{2} & 0 & 0 & 0 & 0 \\
* & * & * & -\Lambda_{3} & 0 & h_{1} P & h_{12} P \\
* & * & * & * & -\varepsilon_{2} I & h_{1} P & h_{12} P \\
* & * & * & * & * & -T_{1} & 0 \\
* & * & * & * & * & * & -T_{2}
\end{array}\right]<0
$$

are satisfied. Then, there exists a Luenberger-type observer (5) with observer gain matrix $L=P^{-1} X$ such that the stateestimation error e asymptotically converges to the origin.

Remark 9. The outcomes of Corollaries 7 and 8 demonstrate that the derived results in Theorems 3 and 4 are applicable even for linear (see, e.g., [13]) and, particularly, for Lipschitz nonlinear systems, and such linear or Lipschitz nonlinear delay-range-dependent results for the case of measurement delays have not been fully explored so far. The deduced linear results introduce flexibility in the estimation of state vector under the influence of measurement or output delays. The introduced Lipschitz constant extracts the aftermath of the nonlinear term and facilitates the solution of the optimization problem without complexity.

While $h_{1}=0$, Theorem 3 reduces to the following corollary, which provides the delay-dependent observerdesign approach.

Corollary 10. Consider a one-sided Lipschitz nonlinear system (1) satisfying the time-delay bounds given by (2) (with $h_{1}=0$, i.e., $0 \leq \tau \leq h_{2}$ ) and the one-sided Lipschitz condition (3) along with the quadratic inner-boundedness criterion (4). Suppose there exist symmetric matrices $P \in \mathfrak{R}^{n \times n}, Q_{i} \in \mathfrak{R}^{n \times n}$, and $Z_{j} \in \mathfrak{R}^{n \times n}$ for $i=2,3$, and $j=2$, a matrix $X \in R^{n \times m}$, and scalars $\varepsilon_{1}$ and $\varepsilon_{2}$, such that the inequalities

$$
\begin{aligned}
& P>0, \quad Q_{i}>0, \quad Z_{j}>0, \quad \varepsilon_{1}>0, \quad \varepsilon_{2}>0, \quad \forall i=2,3, \quad j=2, \\
& {\left[\begin{array}{ccccc}
P A+A^{T} P+Q_{2}+Q_{3} & -X C+Z_{2} & 0 & P-\frac{\varepsilon_{1} I}{2}+\frac{\alpha \varepsilon_{2} I}{2} & h_{2} A^{T} P \\
-Z_{2}+\rho \varepsilon_{1} I+\beta \varepsilon_{2} I & -\Lambda_{1} & Z_{2} & 0 & -h_{2} C^{T} X^{T} \\
* & -\Lambda_{1} & -\Lambda_{3} & 0 & 0 \\
* & * & * & -\varepsilon_{2} I & h_{2} P \\
* & * & * & * & -T_{2}
\end{array}\right]<0 \text {, }}
\end{aligned}
$$

are affirmed. Then, there exists a Luenberger-type observer (5) with observer gain matrix $L=P^{-1} X$ such that the stateestimation error e asymptotically converges to the origin.

Remark 11. Theorems 3 and 4 provide delay-range-dependent observer-design schemes for one-sided Lipschitz nonlinear systems such that the delay satisfies an interval $0 \leq h_{1} \leq \tau \leq h_{2}$; whereas, in Corollary 10, the traditional delay-dependent approach, assuming the lower bound as zero, is used to obtain interesting and novel delaydependent results as a special category. Hence, the proposed derivation in Theorems 3 and 4, employing an advance delay-range-dependent concept, can be less conservative and more appropriate than the traditionalistic delay-dependent approaches. It is worth mentioning that an advanced delay-range-dependent rather than delay-dependent approach has been exploited in the present work to establish state-estimation strategies. 
By taking $Q_{3}=0$, Theorem 4 reduces to the following delay-rate-independent approach for the fast time-varying delay case.

Corollary 12. Consider a one-sided Lipschitz nonlinear system (1) satisfying the time-delay bound given by (2) with $\dot{\tau} \geq 1$, a one-sided Lipschitz condition (3), and the quadratic innerboundedness criterion (4). Suppose there exist symmetric matrices $P \in \mathfrak{R}^{n \times n}, Q_{i} \in \mathfrak{R}^{n \times n}$, and $Z_{j} \in \mathfrak{R}^{n \times n}$ for $i=1,2$, and $j=1,2$, a matrix $X \in \mathfrak{R}^{n \times m}$, and scalars $\varepsilon_{1}$ and $\varepsilon_{2}$, such that the inequalities

$$
\begin{aligned}
& P>0, \quad Q_{i}>0, \quad Z_{j}>0, \quad \varepsilon_{1}>0, \quad \varepsilon_{2}>0, \quad \forall i=1,2, \quad j=1,2,
\end{aligned}
$$

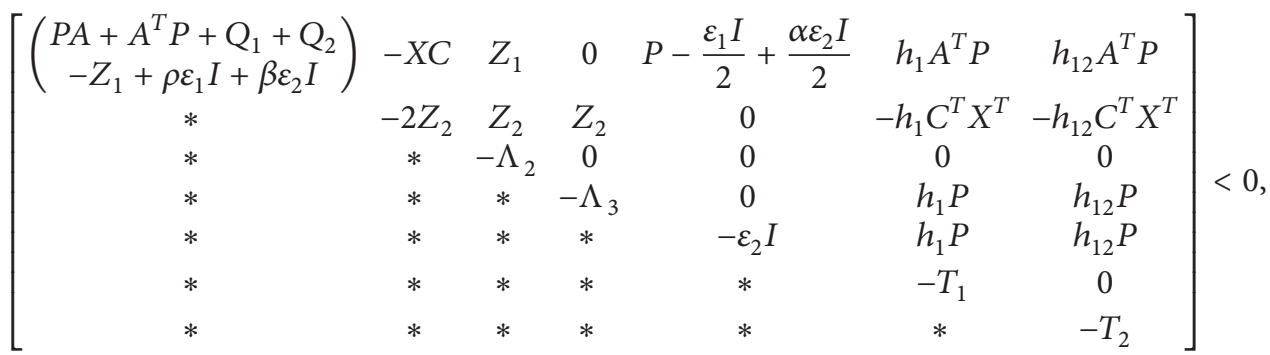

are satisfied. Then, there exists a Luenberger-type observer (5) with observer gain matrix $L=P^{-1} X$ such that the stateestimation error e asymptotically converges to the origin.

Remark 13. Corollary 12 provides a delay-range-dependent stability criterion for the estimation error dynamics (8) under fast time-varying delays. If $\mu \geq 1$, Corollary 12 , instead of Theorem 4, can be effectively utilized to design an observer; consequently, a delay-rate-independent transformation of the delay-range-dependent scheme of Theorem 4 for observer synthesis is operable. Such results as in Corollary 12 are applicable to the nonlinear systems with fast varying delays and are detailed to provide a plausible solution to the observer-design dilemma.

While designing an observer for a nonlinear system (1), a major issue emerged with unknown rapidly varying quantities such as external disturbances (for disturbance rejection see e.g., [48]), due to the fact that a small perturbation can cause the parametric estimates to drift towards infinity. Consequently, an observer for which the stateestimation error can diverge for a small-degree of perturbation is considered as fragile (see, e.g., [49]). Therefore, the scope of Theorem 4 is broadened in Theorem 14 to address the matter of robustness for observer formulation.

Theorem 14. Consider a one-sided Lipschitz nonlinear system (1) satisfying the time-delay bounds given by (2) and a onesided Lipschitz condition (3) along with the quadratic innerboundedness criterion (4). Suppose there exist symmetric matrices $P \in \Re^{n \times n}, Q_{i} \in \mathfrak{R}^{n \times n}$, and $Z_{j} \in \mathfrak{R}^{n \times n}$ for $i=1,2,3$, and $j=1,2$, a matrix $X \in R^{n \times m}$, and scalars $\gamma, \varepsilon_{1}$, and $\varepsilon_{2}$, such that the inequalities (10), $\gamma>0$, and

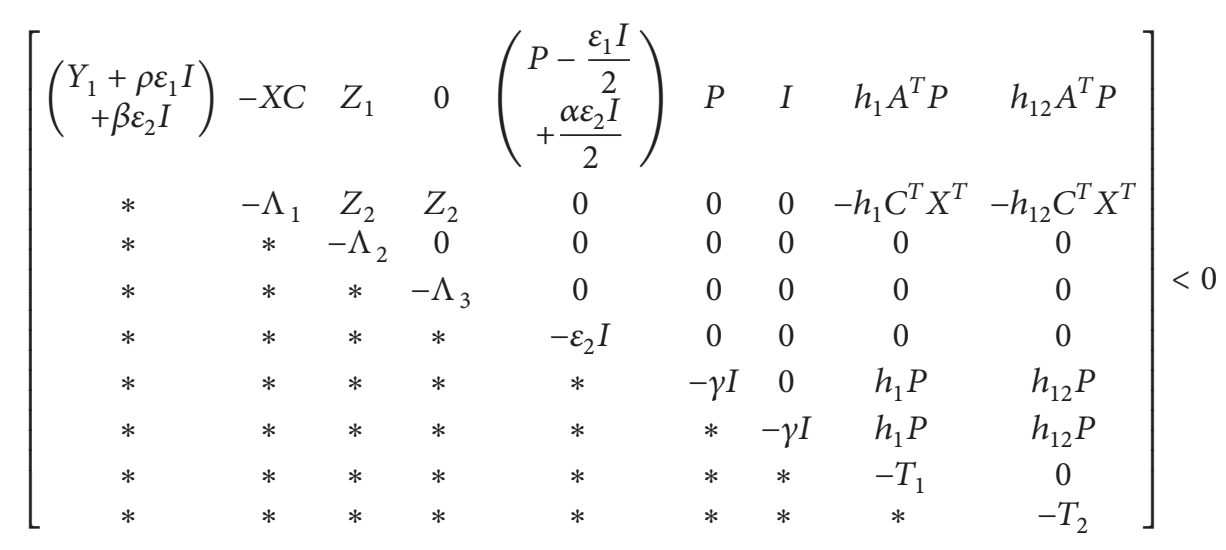

are satisfied. Then, there exists a Luenberger-type observer (5) with observer gain matrix $L=P^{-1} X$ such that the stateestimation error e asymptotically converges to the origin, if $d=0$, and, if $d \neq 0$, the $L_{2}$ gain of the state-estimation error $e$ with regard to the disturbance $d$ is bounded by $\gamma$.
Proof. Consider the LK functional (13). Incorporating the inequality for $L_{2}$ gain reduction

$$
J(e, t)=\dot{V}(e, t)+\gamma^{-1} e^{T} e-\gamma d^{T} d<0
$$


yields

$$
\begin{gathered}
\int_{0}^{t} \dot{V}(e, t) d t+\int_{0}^{t}\left(\gamma^{-1} e^{T} e\right) d t-\int_{0}^{t}\left(\gamma d^{T} d\right) d t<0, \\
V(e, t)-V(e, 0)+\int_{0}^{t}\left(\gamma^{-1} e^{T} e\right) d t-\int_{0}^{t}\left(\gamma d^{T} d\right) d t<0,
\end{gathered}
$$

which further implies

$$
\sqrt{\int_{0}^{t}\left(e^{T} e\right) d t}<\gamma \sqrt{\int_{0}^{t}\left(d^{T} d\right) d t}
$$

That is, $\|e\|_{2}<\gamma\|d\|_{2}$. Considering the case when $d=0$, inequality (32) shows that the estimation error asymptotically converges to zero. And if $d$ belongs to $L_{2}$ bounded perturbations, (34) produces $\|e\|_{2} /\|d\|_{2}<\gamma$ (see also [50,51]). Working out the same procedure, under disturbance $d \neq 0$, as seen in the proof of Theorem 3, we have $J(e, t) \leq \Psi_{2}^{T} \Upsilon_{2} \Psi_{2}$, where

$\Upsilon_{2}$

$$
\begin{gathered}
{\left[\begin{array}{cccccc}
W & -P L C-A^{T} Y_{4} L C & Z_{1} & 0 & Y_{2}-\frac{\varepsilon_{1} I}{2}+\frac{\alpha \varepsilon_{2} I}{2} & Y_{2} \\
* & -\Lambda_{1}+Y_{3} L C & Z_{2} & Z_{2} & -Y_{3} & -Y_{3} \\
* & * & -\Lambda_{2} & 0 & 0 & 0 \\
* & * & * & -\Lambda_{3} & 0 & 0 \\
* & * & * & * & -\varepsilon_{2} I+Y_{4} & Y_{4} \\
* & * & * & * & * & -\gamma I+Y_{4}
\end{array}\right],} \\
\Psi_{2}^{T}=\left[\begin{array}{llllll}
e^{T} & e^{T}(t-\tau) & e^{T}\left(t-h_{1}\right) & e^{T}\left(t-h_{2}\right) & \Phi^{T} & d^{T}
\end{array}\right] .
\end{gathered}
$$

Applying the Schur complement, employing congruence transformation using $\operatorname{diag}\left(I, I, I, I, I, I, I, P Z_{1}^{-1}, P Z_{2}^{-1}\right)$, and defining $X=P L$ and $T_{i}=P Z_{i}^{-1} P$, for $i=1,2$, yield (31), which completes the proof.

Remark 15. Theorem 14, in contrast to Theorem 4, ensures robustness of the observer design for one-sided Lipschitz nonlinear systems subject to time-varying interval time delays under $L_{2}$ norm-bounded disturbances. The results for delay-range-dependent observer design against disturbances are rare, and, in this regard, the proposed methodology for robust state estimation is utilitarian. Moreover, for one-sided Lipschitz dynamical processes, the proposed methodology can be appealing due to its effective utilization in the environment involving perturbations.

The constraints in Theorems 4 and 14 include nonlinear terms contained in $\varphi=\operatorname{diag}\left(-P Z_{1}^{-1} P,-P Z_{2}^{-1} P\right)$, which introduce difficulties in determining the observer gain matrix. The diagonal structure $\varphi$ can be replaced by $\operatorname{diag}\left(-T_{1},-T_{2}\right)$, where $T_{1}=P Z_{1}^{-1} P$ and $T_{2}=P Z_{2}^{-1} P$, to solve the constraints using cone complementary linearization technique $[36,52]$. The original feasibility problem in Theorems 4 or 14 can be solved by optimizing min Trace $\left\{Z_{1} S_{1}+Z_{2} S_{2}+T_{1} \bar{T}_{1}+T_{2} \bar{T}_{2}+\right.$ $P \bar{P}\}$ subject to

$$
\begin{gathered}
{\left[\begin{array}{ll}
P & I \\
* & P
\end{array}\right]>0, \quad\left[\begin{array}{ll}
Z_{i} & I \\
* & S_{i}
\end{array}\right]>0,} \\
{\left[\begin{array}{cc}
T_{i} & I \\
* & \bar{T}_{i}
\end{array}\right]>0}
\end{gathered}
$$

in addition to the constraints in Theorems 4 or 14 as seen in [46], where $S_{i}, \bar{T}_{i}$, and $\bar{P}$ are employed to represent the inverses of $Z_{i}, T_{i}$, and $P$, respectively, for $i=1,2$. Since we have defined $T_{i}=P Z_{i}^{-1} P$, (37) further yields

$$
\left[\begin{array}{cc}
P Z_{i}^{-1} P & I \\
* & \bar{T}_{i}
\end{array}\right]>0, \quad i=1,2 .
$$

Applying congruence transform $\operatorname{diag}(\bar{P}, I)$ and substituting $S_{i}=Z_{i}^{-1}$, the inequality

$$
\left[\begin{array}{ll}
S_{i} & \bar{P} \\
* & \bar{T}_{i}
\end{array}\right]>0, \quad i=1,2
$$

is implicitly obtained. Hence, by including constraint (39) and using $T_{i}=P S_{i} P$ for $i=1,2$, a more appropriate optimization problem than [46] is obtained as

$\min \quad$ Trace $\left(\sum_{i=1}^{2}\left(Z_{i} S_{i}+0.5 P S_{i} P \bar{T}_{i}+0.5 T_{i} \bar{T}_{i}\right)+P \bar{P}\right)$, subject to (36), (37), (39), and inequalities in the Theorems 4 or 14 .

Note that both conditions $T_{i}=\bar{T}_{i}^{-1}$ and $P Z_{i}^{-1} P=\bar{T}_{i}^{-1}$, for $i=$ 1,2 , are given equal weight factors in the objective function of (40).

Remark 16. Recently, a control approach for TS fuzzy systems based on the cone complementary linearization algorithm was developed in [46]. A similar approach by solving min Trace $\left\{Z_{1} S_{1}+Z_{2} S_{2}+T_{1} \bar{T}_{1}+T_{2} \bar{T}_{2}+P \bar{P}\right\}$ subject to (36), (37), and the inequalities in Theorems 4 or 14 can be used to determine observer gain matrix; however, it can lead to conservative results. The inclusion of (39) (or (38)) along with (37) and the application of the modified objective function in optimization (40) ensure $T_{i}=\bar{T}_{i}^{-1}$ and $P Z_{i}^{-1} P=\bar{T}_{i}^{-1}$ for $i=1,2$, which, in contrast to [46], enforces the additional condition $T_{i}=P Z_{i}^{-1} P$ required for obtainment of unique solutions to $T_{1}$ and $T_{2}$ matrices. Hence, constraint (39), defined in the present case, is mandatory in order to obtain a less conservative solution to the nonlinear optimization problem.

Remark 17. The objective function in (40) contains highly nonlinear terms, $P S_{i} P \bar{T}_{i}$ for $i=1,2$. To deal with this 
problem, linearization of the trace function is employed to obtain

$$
\begin{gathered}
\psi\left(S_{i}, T_{i}, \bar{T}_{i}, P, \bar{P}, Z_{i}\right) \\
=\operatorname{Trace}\left(\sum _ { i = 1 } ^ { 2 } \left(Z_{i o} S_{i}+Z_{i} S_{i o}+0.5\right.\right. \\
\times\left(P_{o} S_{i o} P_{o} \bar{T}_{i}+P_{o} S_{i o} P \bar{T}_{i o}+P_{o} S_{i} P_{o} \bar{T}_{i o}\right. \\
\left.\left.\quad+P S_{i o} P_{o} \bar{T}_{i o}+T_{i} \bar{T}_{i o}+T_{i o} \bar{T}_{i}\right)\right) \\
\left.+P_{o} \bar{P}+P \bar{P}_{o}\right)
\end{gathered}
$$

where the subscript o is used to represent the corresponding constant matrices of $S_{i}, T_{i}, \bar{T}_{i}, P, \bar{P}$, and $Z_{i}$, for $i=1,2$, appearing from the linearization process. By application of (41), optimization problem (40) can be solved via available LMItools and the cone complementary linearization algorithm (see details in $[36,52]$ ) to obtain an appropriate solution to Theorems 4 or 14 . The LMI-based proficiencies are preferred owing to their ability in addressing large scale, complex, multiconstraint, and multiobjective optimization problems.

Generally, cone complementary linearization algorithm based solutions for determining controller or observer gains require extra time and computations because of their iterative nature in contrast to the simple LMIs. It should be noted that the observer or controller design conditions like in Theorem 3 for delay-range-dependent systems are hard to convert into LMIs. Additionally, the present work provides an offline solution for the observer gain calculation, for which extra design computations will not affect either feasibility of the constraints or real-time state-estimation application.

\section{Simulation Results}

In this section, we verify the proposed nonlinear timedelay observer-design methodologies using two numerical examples.

Example 18. Consider the dynamics of a nonlinear system borrowed from [16] under delayed output, given by

$$
\begin{gathered}
\dot{x}_{1}=c_{1} x_{2}(t)-l_{1} x_{1}(t), \\
\dot{x}_{2}=c_{2} x_{3}(t)-l_{2} x_{2}(t), \\
\dot{x}_{3}=c_{3} x_{1}(t) x_{2}(t)+c_{4} \cos \left(x_{2}(t)\right)-l_{3} x_{3}(t)+c_{3} u(t), \\
y(t)=x_{1}(t-\tau(t)) .
\end{gathered}
$$

Using the parametric values mentioned in [16], we come up with the model as

$$
\begin{aligned}
& f(x, u)=\left[\begin{array}{lll}
0 & 2 \cos \left(x_{2}(t)\right)+8 u(t) & 0
\end{array}\right]^{T}, \\
& A=\left[\begin{array}{ccc}
-1 & 0.9 & 0 \\
0 & -1 & 0.4 \\
0.48 & 0.3 & -1
\end{array}\right], \quad C=\left[\begin{array}{l}
1 \\
0 \\
0
\end{array}\right]^{T}
\end{aligned}
$$

The output experiences a measurement delay $\tau(t)$ and the input function is $u(t)=\sin (0.35(t))$. For the state vector estimation in the presence of output delay, the gain vector for the observer is calculated as

$$
L=\left[\begin{array}{c}
-2.9070 \\
3.2632 \\
-3.0164
\end{array}\right] \text {, }
$$

from the given eigenvalues of $\bar{\lambda}=\{-0.030,-0.031,-0.032\}$. The parameters for quadratic inner-boundedness inequality and one-sided Lipschitz condition are selected as $\alpha=1.5, \beta=$ -1 , and $\rho=3$, respectively. A comparison of the computed upper bounds on delay, that is, $h_{2}$, for $h_{1}=0$, that ensures state estimation is listed in Table 1 . It can be affirmed that the results of the proposed observer strategy are broader and applicable for a large range of delays in contrast to [16]. It is also notable that the proposed approach can also be employed for a specific interval of time delay with $h_{1} \neq 0$.

Example 19. Consider the dynamics of a moving object in Cartesian coordinates (see $[30,32,47]$ ) described by

$$
\begin{aligned}
& f(x, u)=-\left(x_{1}^{2}+x_{2}^{2}\right)\left[\begin{array}{l}
x_{1} \\
x_{2}
\end{array}\right], \\
& A=\left[\begin{array}{cc}
1 & 1 \\
-1 & 1
\end{array}\right], \quad C=\left[\begin{array}{l}
1 \\
0
\end{array}\right]^{T} .
\end{aligned}
$$

The parameters of the one-sided Lipschitz condition and quadratic inner-boundedness inequality are

$$
\rho=0, \quad \alpha=-100, \quad \beta=-99 \text {. }
$$

In contrast to $[30,32,47]$, it is assumed that the output of the system is subject to a measurement delay of $\tau=0.12 \mathrm{sec}$, and the output is available with $y(t)=x_{1}(t-\tau)$. The goal is to estimate the state vector in the presence of the output delay. By solving the optimization problem (40) for Theorem 14 with parameters $h_{1}=0.05, h_{2}=0.25$, and $\mu=0$, we obtain the observer gain matrix as

$$
L=\left[\begin{array}{l}
0.564 \\
0.080
\end{array}\right],
$$

for $\gamma=450.191$. The results of the proposed methodology in the absence of disturbance are shown in Figures 1-3. It is evident in Figures 1 and 2 that the observer states are converging to the plant states, while, as depicted in Figure 3, the state-estimation errors are converging to zero.

To evaluate the observer performance against disturbances, we select

$$
d=\left[\begin{array}{l}
0.2 \sin (12 t) \\
0.15 \cos (8 t)
\end{array}\right]
$$

Figure 4 plots the state-estimation errors against perturbations. It is notable that the estimation errors are converging in a region neighboring the origin, indicating the observer's robustness against time-varying disturbances. 


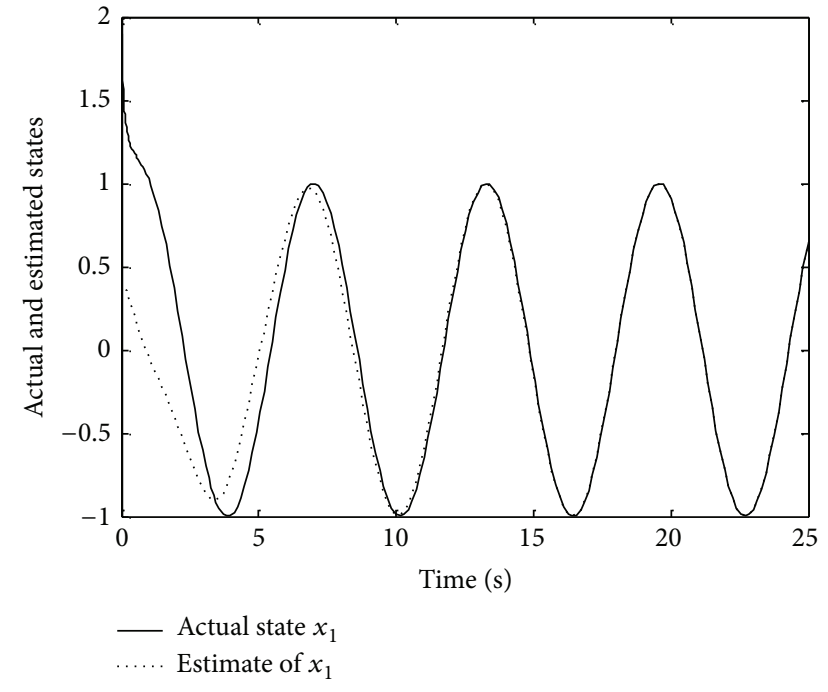

FIGURE 1: Estimation of the plant state $x_{1}$ under measurement delay.

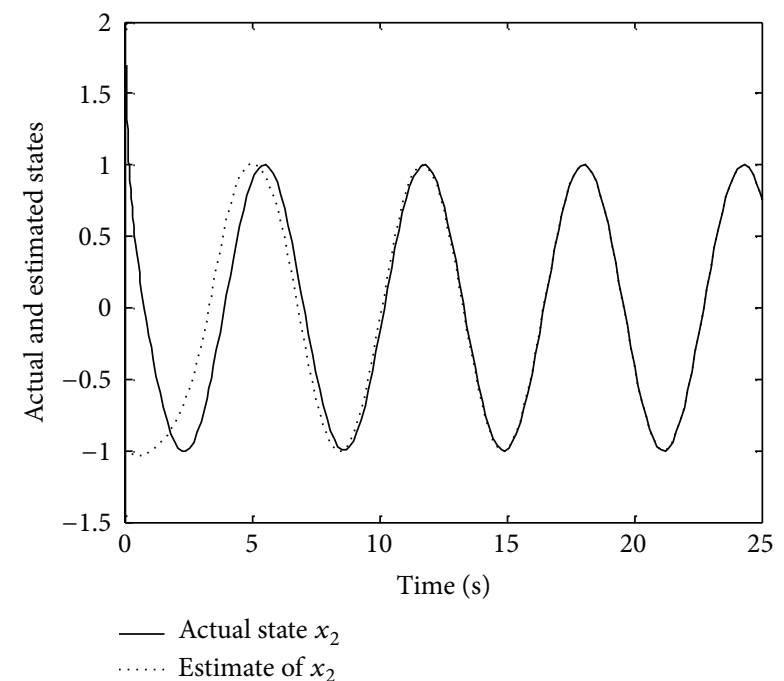

FIGURE 2: Estimation of the plant state $x_{2}$ under measurement delay.

TABLE 1: Allowable upper bound of $h_{2}$ for $h_{1}=0 \mathrm{~s}, \alpha=1.5, \beta=-1$, and $\rho=3$.

\begin{tabular}{lcc}
\hline Methods & $\begin{array}{c}\text { Observer-design approach } \\
\text { in [16] }\end{array}$ & $\begin{array}{c}\text { The proposed } \\
\text { methodology in } \\
\text { Theorem } 3\end{array}$ \\
\hline$h_{2}$ & $16 \mathrm{sec}$ & $\approx 10^{11} \mathrm{sec}$ \\
\hline
\end{tabular}

Focusing on the conservatism of the traditional LMI approaches, the state observer methodologies [30, 32, 47] did not consider time delays; therefore, their applicability to timedelay systems is doubtful. For instance, in [32], the observer gain matrix for the delay free system (45) is found to be

$$
L=\left[\begin{array}{l}
0.0079 \\
0.2661
\end{array}\right]
$$

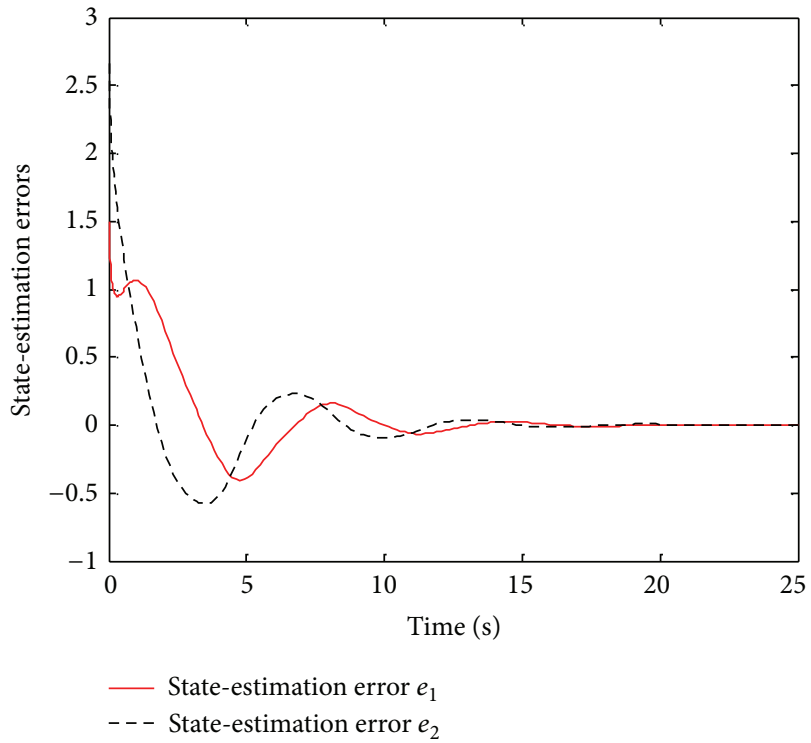

FIGURE 3: State-estimation errors converging to zero by application of the proposed observer strategy against output delay.

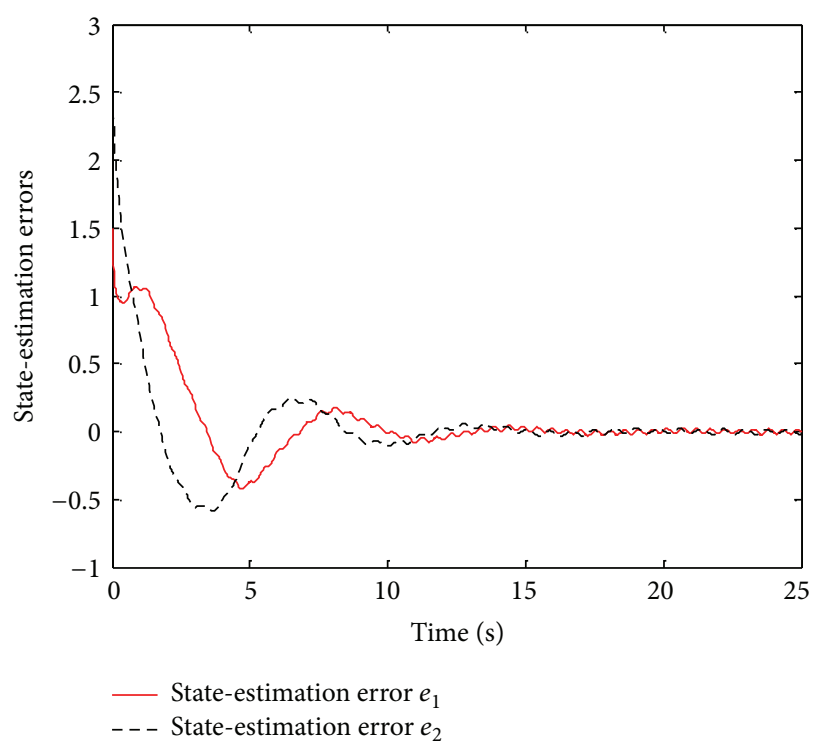

FIGURE 4: Robust state estimation using the proposed observer against disturbances and measurement delay.

With this observer gain, the LMI condition of Theorem 3 is found to be infeasible for a delay range between $h_{1}=0.05$ and $h_{2}=0.25$. Contrastingly, the proposed approach in Theorems 3-14 can be applied for observer synthesis of onesided Lipschitz nonlinear systems subject to measurement delays. It should also be pointed out that the proposed observer gain in (47) cannot be applied for delay free systems because it does ensure stability of error dynamics; however, a suitable observer gain can also be calculated from the proposed approach in Theorems 3-14 by setting $h_{1}=h_{2}=0$. To end, the proposed observer-design methodology can be 
efficaciously applied to state estimation of one-sided Lipschitz nonlinear systems against output delays and perturbations.

\section{Conclusion}

A new approach to the nonlinear observer-design problem for nonlinear systems subject to delayed output measurements was presented in this paper. By application of Jensen's inequality, LK functional, LMI tools, and appropriate matrix transformations, the delay-range-dependent conditions, solvable via the cone complementary linearization algorithm for observer synthesis of one-sided Lipschitz nonlinear systems with time-varying output delays, were derived. An observer construction methodology for estimation of the states of dynamical nonlinear systems that is robust against disturbances was formulated by application of $L_{2}$ stability theory. The methodology is less conventional and more pragmatic than the traditional approaches, due to consideration of unavoidable output delays for observer design of one-sided Lipschitz nonlinear systems. The additional contributions of the present work are the incorporation of delay-range and time-varying delays, as well as the treatment of fast timevarying delays in the system dynamics and nonlinear terms in the design constraints for observer derivation. The resultant observer-synthesis approach can be applied to the estimation of the states of industrial nonlinear systems with outputinterval time-varying delays and disturbances. Simulation results demonstrated the tractability and effectiveness of the projected one-sided Lipschitz observer-design schemes for nonlinear output-delay systems.

\section{Conflict of Interests}

The authors declare that there is no conflict of interests regarding the publication of this paper.

\section{Acknowledgments}

This work was supported by Higher Education Commission (HEC) of Pakistan through indigenous Ph.D. scholarship program (phase II, batch II, 2013) and by the National Research Foundation of Korea (Grant no. MEST-2012R1A2A2A01046411).

\section{References}

[1] S. X. Chen and J. J. Moskwa, "Application of nonlinear slidingmode observers for cylinder pressure reconstruction," Control Engineering Practice, vol. 5, no. 8, pp. 1115-1121, 1997.

[2] A. Iratni, R. Katebi, and M. Mostefai, "On-line robust nonlinear state estimators for nonlinear bioprocess systems," Communications in Nonlinear Science and Numerical Simulation, vol. 17, no. 4, pp. 1739-1752, 2012.

[3] F. Cacace, V. Cusimano, L. Di Paola, and A. Germani, "Observer-based techniques for the identification and analysis of avascular tumor growth," Mathematical Biosciences, vol. 234, no. 2, pp. 147-153, 2011.
[4] C. P. Tan and C. Edwards, "Sliding mode observers for detection and reconstruction of sensor faults," Automatica, vol. 38, no. 10, pp. 1815-1821, 2002.

[5] M. Liu, L. Zhang, P. Shi, and H. R. Karimi, "Robust control of stochastic systems against bounded disturbances with application to flight control," IEEE Transactions on Industrial Electronics, vol. 61, no. 3, pp. 1504-1515, 2014.

[6] M. N. Mahyuddin, J. Na, G. Herrmann, X. Ren, and P. Barber, "Adaptive observer-based parameter estimation with application to road gradient and vehicle mass estimation," IEEE Transactions on Industrial Electronics, vol. 61, no. 6, pp. 28512863, 2014.

[7] H. Rahimi-Eichi, F. Baronti, and M.-Y. Chow, "Online adaptive parameter identification and state-of-charge coestimation for Lithium-polymer battery cells," IEEE Transactions on Industrial Electronics, vol. 61, no. 4, pp. 2053-2061, 2014.

[8] P. S. Teh and H. Trinh, "Design of unknown input functional observers for nonlinear systems with application to fault diagnosis," Journal of Process Control, vol. 23, no. 8, pp. 1169-1184, 2013.

[9] P. Bogaerts and D. Coutinho, "Robust nonlinear stateestimation of bioreactors based on $\mathrm{H}$-infinity hybrid observers," Computers and Chemical Engineering, vol. 60, no. 1, pp. 315-328, 2014.

[10] S. Banerjee and A. K. Jana, "High gain observer based extended generic model control with application to a reactive distillation column," Journal of Process Control, vol. 24, no. 4, pp. 235-248, 2014.

[11] F. Mairet, M. Moisan, and O. Bernard, "Interval observer with near optimal adaptation dynamics. Application to the estimation of lipid quota in microalgae," International Journal of Robust and Nonlinear Control, vol. 24, no. 6, pp. 1142-1157, 2014.

[12] J. W. Wu and K.-S. Hong, "Delay-independent exponential stability criteria for time-varying discrete delay systems," IEEE Transactions on Automatic Control, vol. 39, no. 4, pp. 811-814, 1994.

[13] T. L. Fernando, V. N. Phat, and H. M. Trinh, "Output feedback guaranteed cost control of uncertain linear discrete systems with interval time-varying delays," Applied Mathematical Modelling, vol. 37, no. 3, pp. 1580-1589, 2013.

[14] M. Kchaou, F. Tadeo, M. Chaabane, and A. Toumi, "Delaydependent robust observer-based control for discrete-time uncertain singular systems with interval time-varying state delay," International Journal of Control, Automation, and Systems, vol. 12, no. 1, pp. 12-22, 2014.

[15] X. Li and Z. Xiang, "Observer design of discrete-time impulsive switched nonlinear systems with time-varying delays," Applied Mathematics and Computation, vol. 229, no. 1, pp. 327-339, 2014.

[16] F. Cacace, A. Germani, and C. Manes, "An observer for a class of nonlinear systems with time varying observation delay," Systems \& Control Letters, vol. 59, no. 5, pp. 305-312, 2010.

[17] B. Zhou, Z.-Y. Li, and Z. Lin, "Observer based output feedback control of linear systems with input and output delays," Automatica, vol. 49, no. 7, pp. 2039-2052, 2013.

[18] C. Lin, Z. Wang, and F. Yang, "Observer-based networked control for continuous-time systems with random sensor delays," Automatica, vol. 45, no. 2, pp. 578-584, 2009. 
[19] A. J. Krener and W. Respondek, "Nonlinear observers with linearizable error dynamics," SIAM Journal on Control and Optimization, vol. 23, no. 2, pp. 197-216, 1985.

[20] X. H. Xia and W. Gao, "Nonlinear observer design by observer error linearization," SIAM Journal on Control and Optimization, vol. 27, no. 1, pp. 199-216, 1989.

[21] A. Zemouche, M. Boutayeb, and G. I. Bara, "Observers for a class of Lipschitz systems with extension to $\mathrm{H}_{\infty}$ performance analysis," Systems \& Control Letters, vol. 57, no. 1, pp. 18-27, 2008.

[22] F. E. Thau, "Observing the state of nonlinear dynamic systems," International Journal of Control, vol. 17, no. 3, pp. 471-479, 1973.

[23] R. Rajamani, “Observers for Lipschitz nonlinear systems," IEEE Transactions on Automatic Control, vol. 43, no. 3, pp. 397-401, 1998.

[24] M. Rehan and K.-S. Hong, "Modeling and automatic feedback control of tremor: adaptive estimation of deep brain stimulation," PLoS ONE, vol. 8, no. 4, Article ID e62888, 2013.

[25] U. H. Shah and K.-S. Hong, "Input shaping control of a nuclear power plant's fuel transport system," Nonlinear Dynamics, vol. 77, no. 4, pp. 1737-1748, 2014.

[26] F. Zhu and Z. Han, "A note on observers for Lipschitz nonlinear systems," IEEE Transactions on Automatic Control, vol. 47, no. 10, pp. 1751-1754, 2002.

[27] M.-S. Chen and C.-C. Chen, "Robust nonlinear observer for Lipschitz nonlinear systems subject to disturbances," IEEE Transactions on Automatic Control, vol. 52, no. 12, pp. 23652369, 2007.

[28] M. J. Khosrowjerdi, "Robust sensor fault reconstruction for Lipschitz nonlinear systems," Mathematical Problems in Engineering, vol. 2011, Article ID 146038, 17 pages, 2011.

[29] M. Benallouch, M. Boutayeb, and M. Zasadzinski, "Observer design for one-sided Lipschitz discrete-time systems," Systems \& Control Letters, vol. 61, no. 9, pp. 879-886, 2012.

[30] W. Zhang, H. Su, H. Wang, and Z. Han, "Full-order and reduced-order observers for one-sided Lipschitz nonlinear systems using Riccati equations," Communications in Nonlinear Science and Numerical Simulation, vol. 17, no. 12, pp. 4968-4977, 2012.

[31] Y. Zhao, J. Tao, and N. Shi, "A note on observer design for onesided Lipschitz nonlinear systems," Systems \& Control Letters, vol. 59, no. 1, pp. 66-71, 2010.

[32] W. Zhang, H. Su, Y. Liang, and Z. Han, "Non-linear observer design for one-sided Lipschitz systems: an linear matrix inequality approach," IET Control Theory \& Applications, vol. 6, no. 9, pp. 1297-1303, 2012.

[33] M. Wang, S. S. Ge, and K.-S. Hong, "Approximation-based adaptive tracking control of pure-feedback nonlinear systems with multiple unknown time-varying delays," IEEE Transactions on Neural Networks, vol. 21, no. 11, pp. 1804-1816, 2010.

[34] N. Kazantzis and R. A. Wright, "Nonlinear observer design in the presence of delayed output measurements," Systems and Control Letters, vol. 54, no. 9, pp. 877-886, 2005.

[35] F. Li, P. Shi, L. Wu, and X. Zhang, "Fuzzy-model-based Dstability and non-fragile control for discrete-time descriptor systems with multiple delays," IEEE Transactions on Fuzzy Systems, vol. 22, no. 4, pp. 1019-1025, 2014.

[36] K. Hu and J. Yuan, "Finite sum equality approach to $H_{\infty}$ outputfeedback control for switched linear discrete-time systems with time-varying delay," IET Control Theory \& Applications, vol. 3, no. 8, pp. 1006-1016, 2009.

[37] A. Turnip, K.-S. Hong, and M. Y. Jeong, "Real-time feature extraction of P300 component using adaptive nonlinear principal component analysis," BioMedical Engineering Online, vol. 10, article 83, 20 pages, 2011.

[38] M. Rehan and K.-S. Hong, "Robust synchronization of delayed chaotic FitzHugh-Nagumo neurons under external electrical stimulation," Computational and Mathematical Methods in Medicine, vol. 2012, Article ID 230980, 11 pages, 2012.

[39] F. Li, L. Wu, and P. Shi, "Stochastic stability of semi-Markovian jump systems with mode-dependent delays," International Journal of Robust and Nonlinear Control, 2013.

[40] F. Li and X. Zhang, "A delay-dependent bounded real lemma for singular LPV systems with time-variant delay," International Journal of Robust and Nonlinear Control, vol. 22, no. 5, pp. 559$574,2012$.

[41] S. Zhou and J. Lam, "Robust stabilization of delayed singular systems with linear fractional parametric uncertainties," Circuits, Systems, and Signal Processing, vol. 22, no. 6, pp. 579-588, 2003.

[42] Y. He, Q. Wang, C. Lin, and M. Wu, "Delay-range-dependent stability for systems with time-varying delay," Automatica, vol. 43, no. 2, pp. 371-376, 2007.

[43] H. Shao, "Improved delay-dependent stability criteria for systems with a delay varying in a range," Automatica, vol. 44, no. 12, pp. 3215-3218, 2008.

[44] P.-L. Liu, "Further improvement on delay-range-dependent stability results for linear systems with interval time-varying delays," ISA Transactions, vol. 52, no. 6, pp. 725-729, 2013.

[45] J. Cao, "Improved delay-dependent exponential stability criteria for time-delay system," Journal of the Franklin Institute, vol. 350, no. 4, pp. 790-801, 2013.

[46] C. Peng and Q.-L. Han, "Delay-range-dependent robust stabilization for uncertain T-S fuzzy control systems with interval time-varying delays," Information Sciences, vol. 181, no. 19, pp. 4287-4299, 2011.

[47] M. Abbaszadeh and H. J. Marquez, "Nonlinear observer design for one-sided Lipschitz systems," in Proceedings of the American Control Conference (ACC '10), pp. 5284-5289, Baltimore, Mass, USA, July 2010.

[48] M. Aqil, K.-S. Hong, and M.-Y. Jeong, "Synchronization of coupled chaotic FitzHugh-Nagumo systems," Communications in Nonlinear Science and Numerical Simulation, vol. 17, no. 4, pp. 1615-1627, 2012.

[49] L. Wu, X. Su, and P. Shi, "Sliding mode control with bounded $L_{2}$ gain performance of Markovian jump singular time-delay systems," Automatica, vol. 48, no. 8, pp. 1929-1933, 2012.

[50] M. Rehan, A. Q. Khan, M. Abid, N. Iqbal, and B. Hussain, "Anti-windup-based dynamic controller synthesis for nonlinear systems under input saturation," Applied Mathematics and Computation, vol. 220, no. 1, pp. 382-393, 2013.

[51] M. Rehan and K.-S. Hong, "Decoupled-architecture-based nonlinear anti-windup design for a class of nonlinear systems," Nonlinear Dynamics, vol. 73, no. 3, pp. 1955-1967, 2013.

[52] L. El Ghaoui, F. Oustry, and M. AitRami, "A cone complementarity linearization algorithm for static output-feedback and related problems," IEEE Transactions on Automatic Control, vol. 42, no. 8, pp. 1171-1176, 1997. 


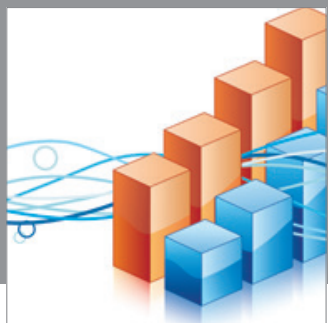

Advances in

Operations Research

mansans

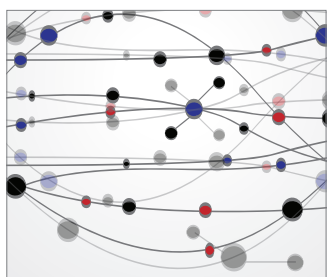

The Scientific World Journal
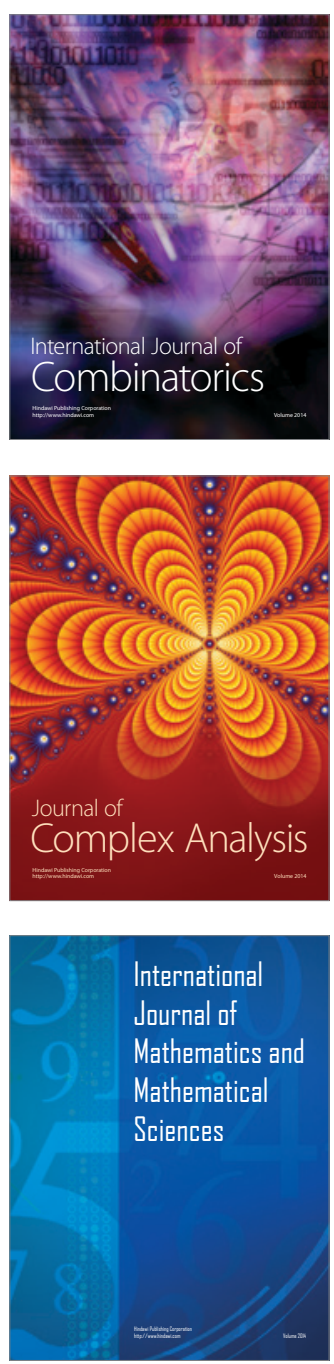
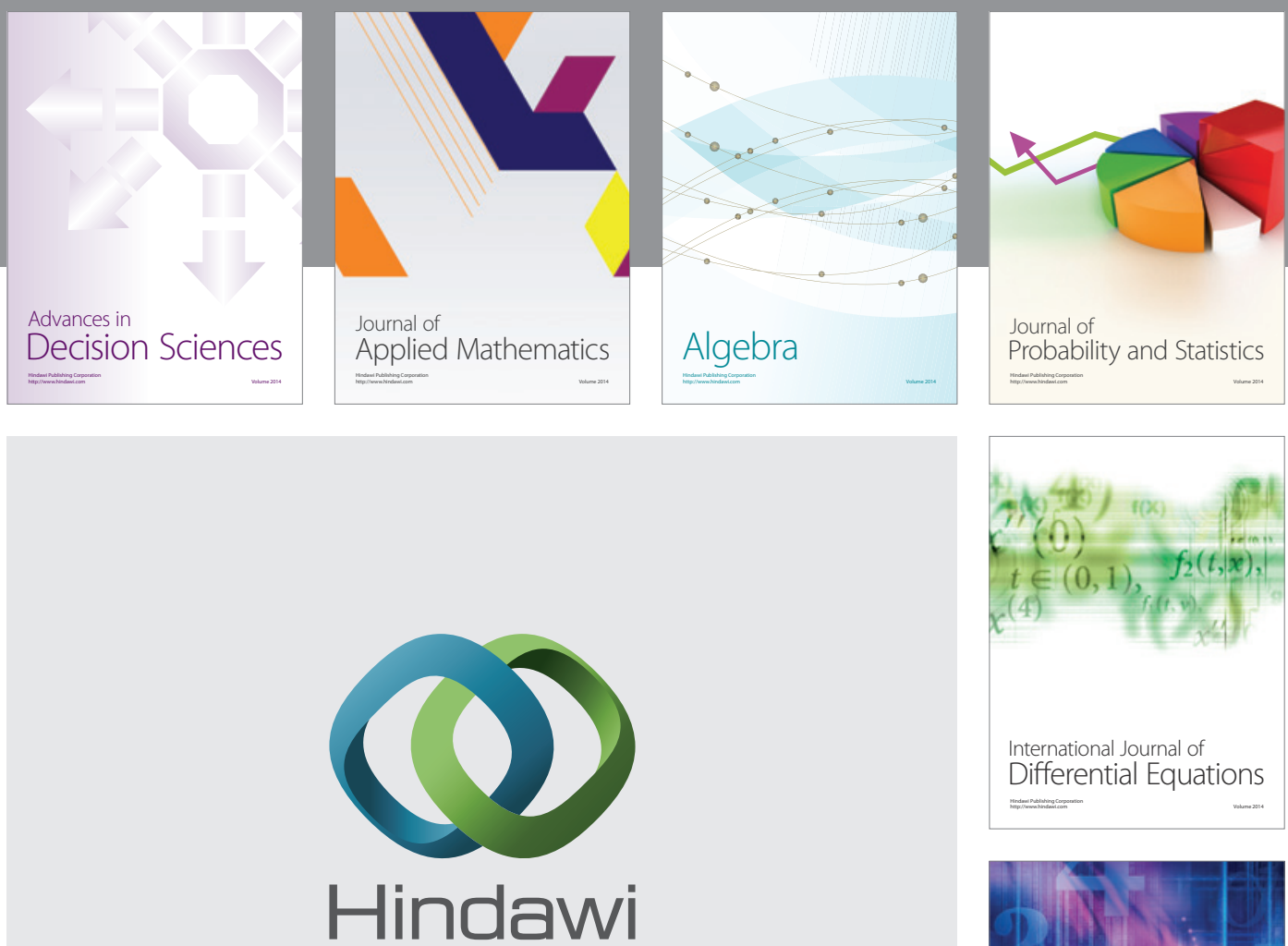

Submit your manuscripts at http://www.hindawi.com
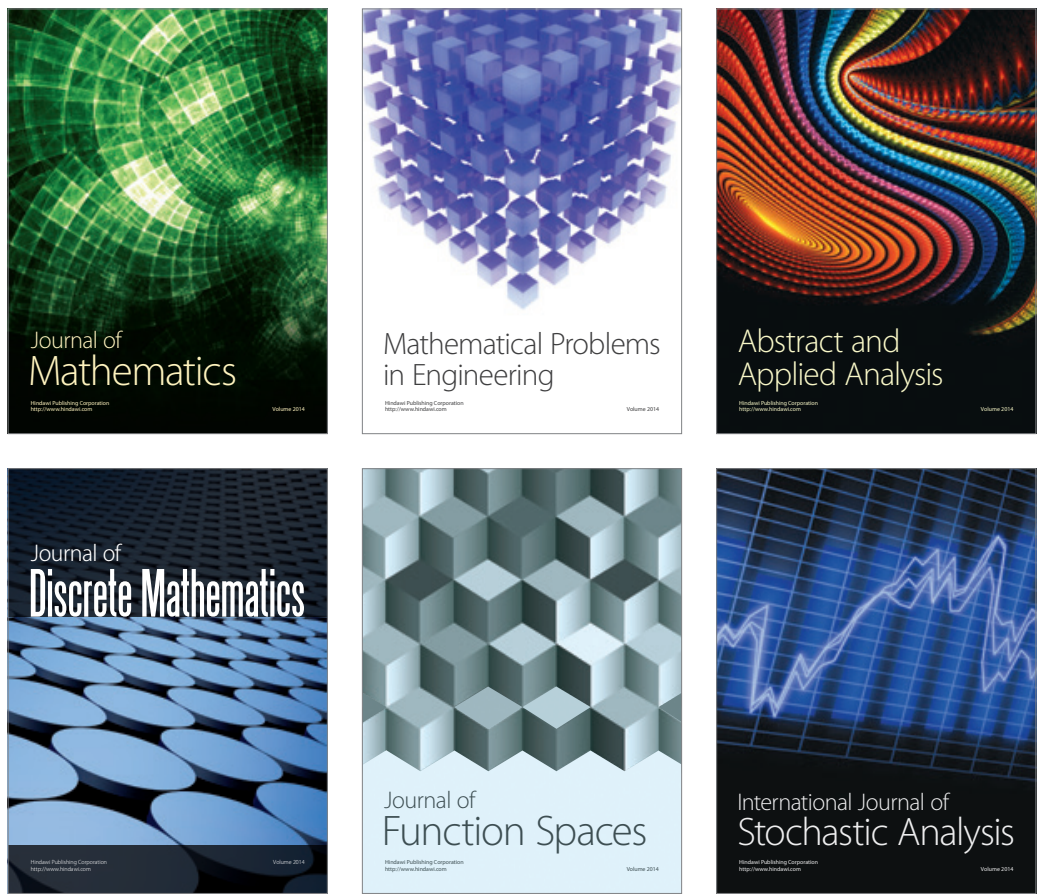

Journal of

Function Spaces

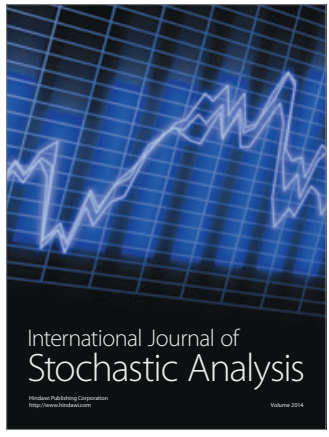

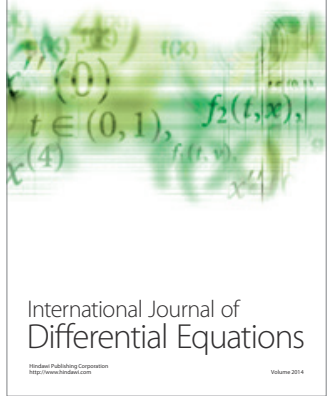
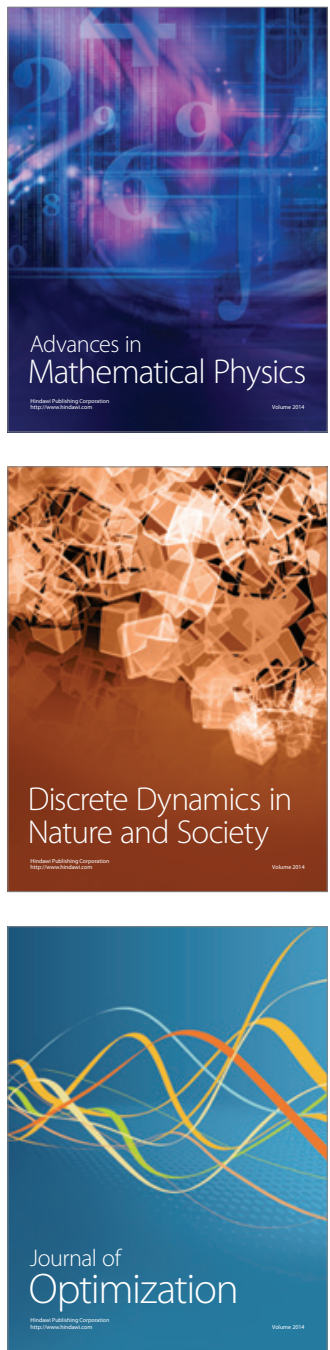